\title{
Adaptive User Interfaces Development Platform
}

\author{
Jing-Hua Ye and John Herbert \\ Department of Computer Science, \\ Kane Building, \\ University College Cork, \\ College Road, \\ Cork, Republic of Ireland \\ $\{$ jhy1, j.herbert\}@cs.ucc.ie
}

\begin{abstract}
Most documents dynamically generated by a web server are in HTML format. However the use of dynamic HTML documents severely limits the amount of user interface (UI) validation that can be done. In order to generalize the abstract UI platform and strengthen the UI validation process, we developed a novel platform to support both UI adaptation and dynamic UI construction. It provides a generic architecture for runtime adaptive UI development based on various XML technologies. As well as making use of different built-in modules, one can extend the platform by adding new functionalities into it. The ease of use of the platform is illustrated using a case study of an on-line accommodation booking form based on the official web site of the English town of Windsor.
\end{abstract}

\section{Introduction}

With the increasing availability of wireless technologies people can now use a variety of hand-held computing devices, such as PDAs, smart phones and pagers to access the same information from any place at any time. Due to varying input/output techniques, modalities, interaction mechanisms, as well as physical constraints, the UIs used to access this information across devices are often drastically different. It is very difficult to build UIs that work across multiple devices without duplicating development effort. The difficulty in designing UIs for multiple devices prompts the need for the development of a multi-device adaptive UI. The goal of this work is to facilitate UI development for devices with restrictive capabilities. An innovative generic software architecture has been developed as a framework. It provides various adaptation mechanisms and provides an XML-based dynamic UI generation mechanism that is a significant feature of the framework.

\section{Software Architecture for Adaptation}

Figure 1 shows the main building blocks of this framework, which has been designed and implemented with an Xalan-XSLT-processor [1], the Apache web server and the JAXP package [2]. 


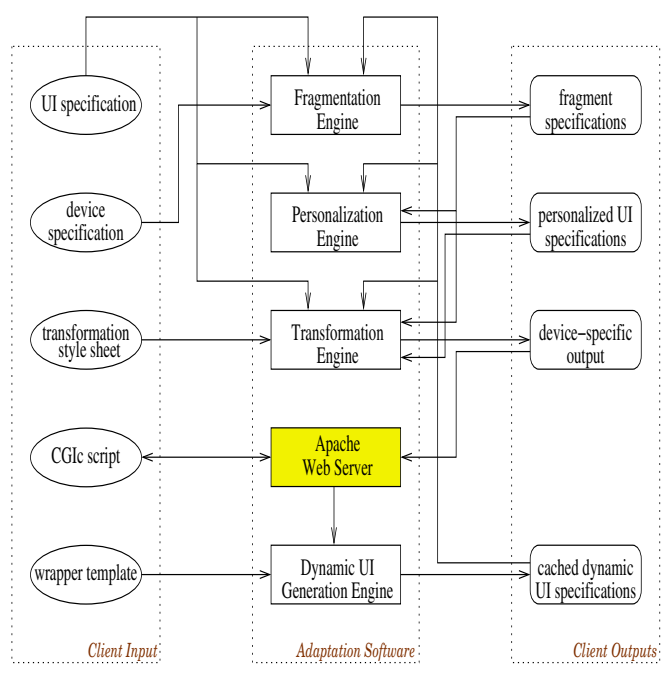

Fig. 1. Generic architecture for a runtime adaptive UI Development System

The UI and device specifications, the associated transformation style sheet, the CGIc script [3], and the wrapper template are required as the inputs to the adaptation software. Each specification is created by UI developers at design time. Developers can use XUL to specify the logical structure of the UI in a UI specification. It is prudent for developers to explicitly specify whether each XUL element can be split or not by giving a yes/no value to the "breakable" attribute of each one. All physical constraints on the target device (i.e. memory capacity, screen size, resolution etc) are specified in a standard XML format. All elements in the UI specification can be transformed to some corresponding device-specific ones by applying the rules specified in an XSL based transformation style sheet. A CGIc script is a standard C program imported from the CGI library. It wraps up user requests submitted via the Apache web server into a standard XML file. A wrapper template is a standard XML file containing a set of named gaps and source code for a target page that will be constructed dynamically.

The fragmentation engine runs a new fragmentation technique 44 to paginate a complex UI by using SAX APIs to index each abstract XUL element in order to minimize the mismatch between the presentation of a UI and a device's capabilities to present it. This fragmentation engine provides developers with the convenience of UI development for multiple devices.

The personalization engine deals with personalizing the adaptive UIs according to the user requests received from the external personalization request handlers. In particular, this engine has adopted a controller-filter architecture [5] so that various types of personalization can be supported by a single module. Link personalization, user-based personalization, and context personalization are supported by this engine.

The transformation engine transforms a UI specification into a device-specific one. The most important feature of this engine is that it supports both the DOM 
and SAX transformations. In addition, the SAX transformation engine uses a special filter pipelining architecture [6] to transform a single page.

The dynamic UI generation engine is based on the wrapper template, which is a standard XML document with named gaps. A new process constructs dynamic XML documents by using a combination of pruning techniques and plug operations for the gaps in the wrapper template. The generated dynamic UI specifications can be further adapted by feeding them back into any one of the engines (e.g. the personalization engine). This pruning-plugging process effectively provides a flexible means of constructing abstract dynamic UIs prior to a validation process.

\section{Evaluation}

To illustrate the ease of using the framework we have implemented a simple accommodation booking form for the official web site of the English town of Windsor (www.windsor.gov.uk). The accommodation booking form available on this site is in a hard-coded HTML format. Consequently, it can only be displayed on regular desktop PCs. However, it would be more desirable for holiday-makers to reserve their accommodations anytime, anywhere with their small screen devices. This problem can be easily overcome with our framework.

We re-coded the Windsor accommodation booking form in the XUL language. This booking form can be adapted to the various needs of UI developers. There are three important possible scenarios for this adaptation. The first scenario is to directly transform the form into HTML format. The second scenario is to paginate the XUL-encoded accommodation booking form into a seven-page PDA presentation. The third scenario is to personalize the XUL-encoded accommodation booking form according to the developer's requests, for example, a developer might wish to remove all the images from the booking form. Besides these three scenarios, a web page can also be dynamically generated by submitting the completed booking form to the web server that is supported in our framework. Due to lack of space, we show the result of the third scenario only in figure 2 .

\section{Conclusions}

This paper presents a general framework that provides an innovative generic software architecture for a runtime adaptive UI development system. An abstract UI can be fragmented into a set of UI fragments that satisfies the constraints of the target device. An abstract UI can also be personalized according to the developer's needs and be transformed into a device-displayable UI. This framework also provides a dynamic UI generation engine that allows one to automatically generate a dynamic XML-based UI. Various functionalities can be easily integrated into this architecture in a modular fashion. This framework allows one to easily update or create a new UI for a new application. One can combine the modules provided in this framework according to one's needs. This framework 
makes available sophisticated technologies for UI adaptation in a re-usable extensible architecture that make it easy for UI developers to design, experiment with, and change an adaptable UI.

\section{References}

1. Apache XML Project: Xalan home page. http://xml.apache.org/xalan-j/index.html.

2. Sun Microsystems, Inc.: The JavaTM Web Service Tutorial v1.2.4. (2003), http://java.sun.com/.

3. Boutell.com: CGIc: An ANSI C library for CGI programming. (August 5th 2004) http://www.boutell.com/cgic/.

4. Jing-Hua Ye and John Herbert: User Interface Tailoring for Mobile Computing Devices. 8th ERCIM Workshop "User Interfaces for All". (28th June 2004), Vienna Austria.

5. Jing-Hua Ye and John Herbert: A Generic Architecture for User Interface Personalization. Proceedings of the Workshop on Environments for Personalized Information Access, Working Conference on Advanced Visual Interfaces (AVI 2004) (May 25th, 2004)

6. Jing-Hua Ye and John Herbert: Framework for User Interface Adaptation. 8th ERCIM Workshop "User Interfaces for All". (28th June 2004), Vienna Austria.

\section{A Windsor's Accommodation Booking Form}

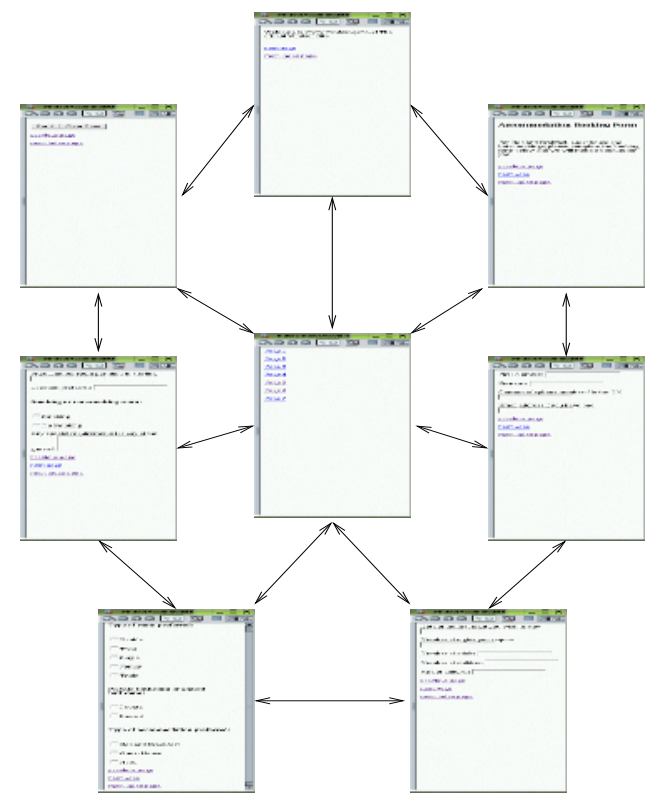

Fig. 2. Fragmented personalized accommodation booking form rendered on a PDA 